\title{
Integration of a Voice Over Internet Protocol (VoIP) Solution with Internet Protocol Version 6 (IPv6) in an Internet Protocol Version 4 (IPv4) Data Network to Increase Employee Productivity \\ A Case Study of Cameroon Telecommunications (Camtel), North-West \\ Regional Representative, Bamenda. Malobe Lottin C.M ${ }^{1}$ \\ The ICT-U Byrd Research Center. Email: malobecyrille.marcel@ictuniversity.org ${ }^{1}$ Phd Student, ICT UNIVERSITY USA - CAMEROON ${ }^{1,2020}$
}

\begin{abstract}
:
Telecommunications organizations have to follow the rapid evolvement of technology especially in the domain of computer information systems and networking if they want to face challenges raised by competition. The goal to respond to the huge market demand of updated products and services from customers requires that the organization's working environment should be equipped with tools and communication facilities that will contribute to ameliorating productivity. This study proposes to integrate a VoIP solution with IPv6 as an alternative communication system that relies on the existing IPv4 data network of Camtel Bamenda. The purpose is to offer to workers a subsequent option that will free them from the poor Quality Of Service (QoS) of their existing Public Switched Telephone Network (PSTN). The first part of this study reports the results of the evaluation of how much such a solution can enhance workers' productivity. The second part describes practically a simulation environment where various configurations of network entities are done following a Dual-Stack transition approach. The data collection method used is open-ended and semi-structured interviews. Also, documentation and records were used to gather information related to the structure, operations, and topological update of the existing IP data network of the organization. The findings indicated that VoIP can be an effective communication solution for Camtel North-West and its implementation with IPv6 will be preferable. However, for this to be efficient there must be a provision of sufficient bandwidth and usage of types of equipment and transmission mediums that minimizes processing and propagation delays.
\end{abstract}

Keywords: Dual-Stack, IPv4, IPv6, VoIP.

\section{INTRODUCTION AND BACKGROUND}

The main focus of the network for any successful integration project is to provide compatibility and interoperability between the existing and the new services and applications (Macgregor, 2015). When a computer network is capable of supporting both data and voice with various other protocols, it is considered to be an integrated network. The simplification and the automation of business processes to the greatest extent possible within a single organization will require that integration should be done following coordinated processes such as the Enterprise Application Integration (EAI) process. The main purpose of EAI is to work as a middleware for supporting and integrating all business processes, internal and external (Farrukh, 2014) while avoiding making sweeping changes to the existing applications or data structures. The understanding of the enterprise architecture is the only way to establish relation regarding how the various domains of this architecture (Technology domain, Applications domain, Data domain, and Business domain) operates and inter-relate with each other. This is an assurance that integration pattern such as mediation (intra-communication between different service /applications/protocols) and federation (intercommunication from different services/applications/protocols) of the new solution can be possible and that the desired goal to improve the communication processes of employees by making use of networks features that enhance productivity within the organization and beyond can be satisfied.

Every computer or device that needs to be connected to the internet or share information in a network independently of the type or size of that network in order to make use of communication facilities must be identified by an address, something that differentiates it from other devices. Besides the 48-bit size Mac addresses that are allocated to each network card interface (burned into the adapter ROM of the Network Card Interface) of a network element, and which uniquely identifies that network element in Ethernet local area network (Layer 2 of the OSI model), some addresses go beyond the local area network and which provides further connectivity to the network elements. These are Internet protocol (IP) 
addresses. After several revisions of the Internet Protocol initially part of the early 1970's Transmission Control Protocol (TCP) version which was later split into two separate protocols, making IP the main communication protocol of the network layer in 1971 while TCP remains in the transport layer), the growth of the Internet has prompted the validation of the official version of this protocol which is the Internet protocol version 4 (IPv4) defined since in the Request for Comment (RFC) 791. This justifies the choice of the researcher to consider this particular version as a base, a foundation of inter-connectivity that must be acknowledged and understood, to consider a possible solution of integration in an IP data network.

Besides other versions of IP, this research also considers the version 6 of the IP protocol (IPv6), a new version of the Internet protocol that brings features that aim to solve limitations observed with IPv4. The changes are from the structure to the operation of the previous version by increasing the IP size so that there is more support of addressable nodes with more flexibility in term of Flow management and security for better Quality of Service (QoS).

Because each device connected on the internet has a usable IP address, this makes the migration of IPv4 to IPv6 look impossible especially because almost all organization that are involved in the competition of market occupation and customer satisfaction makes use of the internet as one of the most important communication platforms and so would not like to see their network experiencing downtime. It then becomes capital to find a way to make these two protocols operate in the same environment or integrate IPv6 in an existing IPv4 network without affecting ongoing operations; especially with the consideration that IPv6 was specifically designed to derive from IPv4. It is in this regard that approaches such as the dual-stack, the tunneling, or the 6-bone are been developed with the main aim to provide hosts, network entities with IPv4 and IPv6 protocol Stack (Gilligan, 1996).

Internet Protocol no matter the version is designed to integrate various types of services such as VoIP. That can justify why today, over the same data network, it is possible to transfer quality digitalized voice signals, video, and audio signals via services such as Voice over IP (VoIP), Television over IP (ToIP), or Radio over IP (RoIP). VoIP, has significant appeal for the enterprise, for service providers, and end-users because it allows the Internet and commonplace data networks, like those at offices, factories, and campuses, to become carriers for voice calls, video conferencing, and other real-time media applications (Wallingford, 2005).

It is therefore important to provide an environment where IPv4 and IPv6 networks and applications /devices can interoperate in the context of VoIP. This environment is based on an architecture that integrates all the components (whether they support IPv4 or IPv6 or both) thereby providing ubiquitous access to IP telephony services.

Statement of the Problem: During the life cycle of an organization and no matter their size, there is a period where the challenge characterized by poor internal communication mechanisms or an unsatisfactory internal communication system becomes a major call of concern. This dissatisfaction is more important when companies realize that the only way through which they get to pass information between one another is either unstable, or unreliable, or insecure. The impact of such a problem is frequent communication breakdowns between entities of the organizations. This can be explained by poor design, poor implementation or just by limited nature of the communication system put in place; so by affecting the organization productivity at large and employees who are the major actors of productivity in particular. The Public Switched Telephone Network (PSTN) or the traditional circuit-switched network is a practical example of a communication system that is widely used and generally accepted as a standard for communication for businesses and households. However, despite the major benefit of the availability of dial tone even when there is a power outage at the customer/end station premises, the PSTN system today does not respond anymore to the pressure that organization face especially regarding the boom of new services and applications that are introduced with the advent of digitalization, the need to go for communication systems that offer less loss of service and better security during the exchange. The main technology that distinguishes Camtel from other telecommunication operators is their PSTN. When this network becomes unstable (ON and OFF tone availability, Noise on the line, Crosstalk during an exchange between parties, Low tone due to under-voltage transmitted on the line), it does not only affect workers but also all the customers sharing that same network. This raises the problem of reliability, privacy control, poor productivity (workers or clients not able to reach each other on time and effectively, delay in procedures and impact in decisions making), with security issues in terms of preserving the integrity of information related to the business and the users of that network. So relying only on this network for the exchange of critical information is a problem that can be addressed by putting into place an alternative digitalize solution that may be dedicated when it comes to exchanging critical information between workers locally and remotely. Besides, The Data network architecture of Camtel is entirely deployed over IP version 4 (IPv4). It means all applications and services used to provide corporate and customer services are designed over IPv4. This constitutes a major problem considering the emergency raised by the IANA (Internet Assigned Number Authority) to the various RIRs (Regional Internet Registry) regarding IPv4 exhaustion of IP addresses. The response to this major problem could be to integrate IPv6 that breaks such barriers and solve other problems raised by the attempt to complement the limitations of IPv4. So, the implementation of a telephony service like VoIP will permit not only to ensure an alternative solution to the PSTN independently of its availability but to also give the users the flexibility to communicate over a more reliable IP data network using the existing corporate data network. The challenge ahead of the researcher regarding these problems is to find out how to prioritize IPv6 traffic over the IPv4 and make VoIP operate over IPv6? Or just deploy both protocols and integrate VoIP with a focus on traffic prioritization of IPv6 over IPv4 and Voice over Data?

\section{Purpose of the Study}

This study investigates the communication working environment of workers of Camtel North-West region with a focus on two communication network platforms: the IP data network and the PSTN. It utilizes the IPv6 version of the internet protocol and determines how to use this version to integrate a digital telephony system that works together with the PSTN so that employee productivity can be improved. 
Specifically, this research proposes to CAMTEL North West Region:

1. An approach they may use to integrate the IPv6 addressing scheme in collaboration with their existing IPV4 data network;

2. A way they may incorporate a VoIP solution in that secure corporate data network to develop another way of communication that frees them from the dependence of the Public Switched Telephone Network.

3. Propose a working IP data environment where IPv4 and IPv6 are used to efficiently run all corporate services and applications while integrating a new service such as VoIP and maintain good QoS of existing services and applications.

Research questions: In the course of our research, the questions raised are:

1. How to integrate IPv6 in the existing IPv4 data network of Camtel North-West?

2. Will this integration affect the existing quality of service? And how to maintain the acceptable Quality Of Service (QoS)?

3. How to integrate a VoIP system that will provide telephony services in the existing IPv4 data network and give priority to IPv6 traffic forwarding?

4. Will this new solution effectively constitute an alternative communication system to the PSTN?

5. How to address delays issues as voice signals are not delayed tolerant?

Significance of the Study: The result of this research will be of great benefit to the following:

- IP data networks of mediums and large organizations in particular, and Internet Service providers in general: today's networks, especially Next-Generation Networks, are characterized specifically by the ability to deliver multimedia content most efficiently to end-users following a convergence design. This is justified by the availability of high bandwidth allocated to forward IP data packets according to the type of service provided. Internet service providers (ISP) like Camtel that provide High bandwidth services such as IPTV (Television Over IP) for example are quite conscious of the fact that Multimedia traffic streams have high bandwidth requirements.

- Public and Private organizations that need alternative secure communication systems and applications: the research make use of the case study of Camtel and previous work done in this domain (IP telephony), to present to the public and private organizations a digital communication technology that they can use together with their existing PSTN infrastructure. The PSTN despite the benefit provided, can no more support the constraint imposed by the advancement in technology related to communication services. This research presents and shows how to integrate VoIP services in the data network of Camtel so that they can have an alternative communication system that is far cheaper to implement, thus providing efficiency in communication and flexibility in the way workers communicate.

- Computer network Designers: the result of this work can be of great use to network designers. It will contribute a great view on how they can design a network on IPv6 and deploy various reliable and secure services and applications.

- Cameroon telecommunications at large and Camtel North West in particular: this research can be very significant to Camtel by showing them a vision on how they can integrate a VoIP solution in all their various regionals networks. It gives to the IP data engineers a good understanding of what benefits IPv6 can contribute to their IP services subscribers (Internet, IPTV, xDSL, etc.) QoS. It also gives to the administration another perspective to improve communication systems of the working environment so to enhance productivity.

- $\quad$ Students and Researchers: the research can also be significant to the student that needs to know what IPv6 is all about and what makes it more interesting than the previous version. It gives to a researcher a global view of the new addressing protocol especially because most organizations are still to migrate towards IPng (IP next-generation) or deploy services such as VoIP in a data environment that is both made of IPv4 and IPv6.

\section{Scope of the Study}

The scope of the study is addressing the IP data network of the regional representation of Camtel and does not in any way consider the physical nor the logical topology of extended regions: the research does not consider all the other 9 regional offices of Camtel. However, it proposes solutions should in case this system needs to be deployed in all the other regions. The study does not emphasize in operation or functionalities of the public Switch Telephone network. Rather, the study addresses the limitations of this system to justify why and how VoIP contributes to solving most of these limitations costeffectively and optimally. The implementation aspect of this solution in this research work is done in the form of simulation and includes the configurations of Cisco IP and Soft Phones, Cisco layer 3 switches and Routers. We shall not consider how configurations apply on extended gateway or gatekeepers are done to provide VoIP services.

\section{LITTERATURE REVIEW}

James (2015) discussing on how Information Technologies services can be integrated and managed in his white paper, thinks that it is the increasing complexity of the IT value chain and the rise of Multi-vendor supplier eco-systems that has led to the rise of service integration and management. These rises contributed to motivating the desire for ends-users to communicate using the latest technologies and consequently prompted telecommunications companies to always perform what we call -IT technological watch (PowerNext, 2016). The response to this desire could not be possible without the ability of organizations to perform systems integration and management in their IP data networks. Systems integration is responsible for getting solutions, different technologies, applications, and infrastructure to work together, with a focus on technology integration (James \& Holland, 2015). Service Integration is ensured by the flexibility offered by the Internet protocol format which facilitates the integration of new services making this protocol a predominant mean for modern data communication. IP offers reliable/unreliable means through which different types of networks running various types of services can transfer different types of data to different destinations. Besides these various services and technologies integrated into the Internet Protocol networks, Internet telephony is exploding, with popular applications such as Skype and others enabling wideband voice and video conferencing over the Internet. This has been made possible with solutions such as voice over IP (VoIP), which happened to revolutionize the telecommunications industry, as circuitswitched equipment from PBX (Private Branch Exchange) to 
long haul equipment is being replaced by soft IP switches. Now that the analogue-to-digital revolution is nearly complete, the world is undergoing an all-media-over-IP revolution, with radio, television, telephony, and stored media all currently being delivered over IP wireline and wireless networks (Schaar \& Philip, 2011). However, according to Floriano (2010), variations in network conditions can have considerable consequences for real-time multimedia applications and can lead to unsatisfactory user experience. This is because Multimedia applications tend to be delaysensitive, bandwidth intense, and loss-tolerant. It is therefore all about finding the best deployment approach that can satisfy the requirement of delay-intolerant multimedia applications and services such as VoIP. In the IEEE Standard Dictionary, a Protocol is defined as a set of rules that govern functional units to achieve communication. (Jarvin, 2004) said in the context of data communication, a protocol is a formal set of rules, conventions, and data structure that governs how computers and other network devices exchange information over a network. In other words, a protocol is a standard procedure and format that two data communication devices must understand, accept, and use to be able to talk to each other. Peter Dordal (2020) thinks that the Internet Protocol was developed to solve the scaling problem with Ethernet and to allow support for other types of LANs and Point-to-Point links as well. He argues that perhaps the central issue in the design of IP was to support universal connectivity (everyone can connect to everyone else) in such a way as to allow scaling to enormous size. The Internet Protocol (IP) is a network-layer protocol that contains addressing information and some control information to enable packets to be routed in a network. IP is the primary network-layer protocol in the TCP/IP protocol suite which represents the heart of the Internet protocols and offer two main functions providing connectionless, best-effort delivery of datagrams through a network with option of fragmentation and reassembly of these datagrams with different maximum-transmission unit (MTU) sizes (Jarvin, 2004). Kiran (2016) identifies 5 majors 'domains where the Internet protocol will face serious challenges in the coming future. These are: Network addressability (a need to provide sufficient public IP addresses to any digital equipment that needs to be connected to the internet), transport (a need to offer more reliable communication sessions to various services and applications), security (a need to keep an eye on - Who access What $\|$ and from where!), network operations (a need to handle massive endpoints, all/anything network-connected) and Mobility requirements(IP needs to achieve the Ideal mobility solution by supporting Optimal Routing). Besides these challenges, Charles (2012) for example in his article "future Internet Protocols", and talking on the future challenge ahead of IP, thinks that besides the problems caused by the exploding address space, the internet protocol needs to have better control over the Quality Of Service (QoS)and for larger packets. He continues by saying that one major challenge is the Internet of Things (IoT) which envisions that everything that has power will connect to the Internet, will drive growth for years to come. Andrew Kirch (2015) in his article - Let the Sky Fall discussing challenges ahead of IPv4 said - The other problem with IPv4 is Network Address Translation (NAT). Overload NAT (one IP with multiple private IPs behind it) breaks quite a few applications and provides no additional security against internet threats. Other issues reported such as Increase prices of Public IPv4 (Heficed, 2019) and Weak Protocol extensibility. To bring solution to these challenges, National Academic Press (2000) believes that it is all about developing protocols for user authentication and protection of messaging traffic. He argues that the Security challenge ahead of IP requires the deployment of a better version of the Internet Protocol such as IPsec. Other solutions have been put in place to try as much as possible to face the future challenges ahead of Internet protocols. For example, there is a solution related to the optimization of the Quality Of Service (QoS) such as Diff-Serv (Differentiation Service). As the name implies, DiffServ allows ISPs (Internet Service Providers) to offer users a range of qualities of service beyond the typical best effort (Blake et al, 1998). Although diff-serv is an improvement over best-effort services, it has several limitations that might preclude its use for some healthrelated applications (Clark \& Wroclawski, 1997). The response to these challenges appears to be the development of a new protocol like the IPng (IPv6). This may justify why Peter (2018) in his article "On the dual-Stacking transition to IPv6: forlon Hope?" thinks that the assumption that IPv6 will inevitably replace IPv4 as the dominant network layer protocol on the internet is almost universal. Nick Buraglio 2018) in his article "Three Reasons why IPv6 is worth the effort" mentioned three main features which he believes are the killer apps or amazing features brought by IPv6. These are End-to-End connectivity, minimize network complexity (with a mechanism such as NAT that is not necessary with IPv6) and ability to make support and content a commonplace (IPv6 has far more support in hardware, software, and content that was available even some years back). This can surely justify why most organization today are migrating towards IPv6. Molay Ghost (2017) reported in his article "Reliance Jio boots India past $20 \%$ IPv6 capability" that the most successful IPv6 deployment thus far has connected almost half a billion people in India. Another organization that succeeded in the deployment and implementation of IPv6 in their existing IPv4 data network is CISCO. Cisco IT (2013) said the migration started with a planning phase that was conducted base on some keys guidelines such as making sure that ongoing processes should not be disrupted or interrupted, new processes should be developed to support operational efficiency, the design should consider coexistence with IPv4 in a long term, the Service Level Agreement (SLA) and security in case of using the Dual-Stack transition technic must be at least as efficient as the existing IPv4. Verizon Wireless, another example, deployed IPv6 in a Dual-Stack mode because of the need to maintain IPv4 traffic for those servers that will continue to use that standard and, IPv6 for newly deployed servers. This Dual-Stack applies both for existing and new Verizon customers (Verizon, 2020). Internet Society (2018) stated that IPv6 deployment continues to increase around the world. The levels of IPv6 deployment in networks and service providers all over the globe have increased dramatically. Over $25 \%$ of all Internet-connected networks advertise IPv6 connectivity and Google reports 49 countries to deliver more than $5 \%$ of traffic over IPv6, with new countries joining all the time. Africa is still very behind and Cameroon especially hasn't yet reported officially any IPv6 traffic towards the World Wide Web. Internet Society (2018) believes that more work is required to drive IPv6 deployment in countries especially for some of the major industrialized nations like Russia, China, Spain and Italy that have not seen significant levels of IPv6 deployment to date. The introduction of IPv6 has opened up many questions concerning its conversion and transition from IPv4 to IPv6; 
this is one critical problem that is being commonly discussed in the networking community nowadays. DoD HPC (2020) mentioned that IETF RFC 7084 and an update to it describe several IPv6 transition mechanisms. Auben networks (2014) categorize these migration techniques in three categories which are Dual-Stack (IPv4 and IPv6 coexist in the same devices and networks. These methods imply the use of both protocols in parallel), Translators (Translation from IPv4 to IPv6 entities and from IPv6 to IPv4 entities to allow the communication between them) and Tunneling (Transport of IPv6 packets in IPv4 tunnels and IPv4 packets in IPv6 tunnels). IPv6 appears to be the designed protocols for multimedia services. Wikiwand (2019) discussed how to provide Multimedia services and elaborate on the IP Multimedia Subsystem or IP Multimedia Core Network Subsystem (IMS). The next-generation network (NGN) of telecommunication organization should be highly performable, expandable, operational, administrable and secure, to realize the features and functions such as highquality streaming media, wireless and mobile capacity, diversification of terminal types of equipment, quality control of on-demand transmission, higher safety and security. IPv6 is commonly agreed to be one of the key technological supports to NGN (Lian et al., 2008). Organizations have been attempting to deploy a VoIP solution using IPv6. Most organization that attempted such a deployment reported that it was a good option. This is why Daniel (2019) thinks that compared with IPv4, IPv6 can deliver more comprehensive communication services to network applications. This requires that Signaling devices and Protocols(H.323/SIP, RTP, RTCP) with CODECs should have IPv6 Capability to be able to implement VoIP in a Dual-Stack Network. Even though Finnell (2018) believes that PSTN is viewed as more reliable and secure than VoIP networks, he admitted that VoIP services have improved and said VoIP has its advantages, including lower network infrastructure costs, scalability, and advanced features, such as unified communications and applications integrations. Integration should be in consideration of fundamental parameters such as latency (ranges from 20 to $150 \mathrm{~ms}$ : the maximum acceptable latency allowable in a VoIP data network is $250 \mathrm{~ms}$. Above this value, there is a serious drop in the quality of voice traffic) (Unuth, 2019), Jitter (The low tolerance and acceptable jitter value is $30 \mathrm{~ms}$. At $100 \mathrm{~ms}$, jitter becomes a serious Quality Of Service issue), bandwidth (Consideration of symmetric minimum bandwidth of $100 \mathrm{kbps}$ (up and down) is advisable for 1 concurrent call)(Kimball, 2015), Packet Loss (10\% packet loss will cause serious poor quality because of the real-time nature of IP telephony), (Titus, 2019), Reliability and Security (It means providing a system that is $99.9 \%$ up together with a good call quality of voice exchange in the most secure way and considering implementing call encryption to preserve VoIP privacy and protect the vulnerabilities of the system) (Cameron, 2020).

\section{RESEARCH METHODOLOGY, DATA ANALYSIS AND EVALUATION OF THE EXISTING SYSTEM}

\section{RESEARCH METHODOLOGY}

This research follows a Qualitative and Applied research methodology. The methodology helped in finding:

How much VoIP will enhance workers' Productivity? The research design is considered here as a case study.
Define an approach of how such a solution can be integrated into an existing IPv4 data network with IPv6. To achieve this research goal, the researcher followed an engineering research Process that helped in identifying the problem, conduct related research background concerning research questions, define requirements for the implementation of IPv4/IPv6 solutions, Brainstorm on the possible design about Dual-Stack, Translation and Tunneling mechanisms, validate a design and conduct test to see effectively VoIP operation in a Dual-Stack network architecture.

The study used a deductive approach where the Alternative Hypothesis (Ha) confirmed True: "The Integration of VoIP with IPv6 in the IPv4 data network of Camtel North-west will improve the communication systems of workers and by so, making them more productive".

". The relation between the keycodes generated from the responses of interviewees and the productivity is strong and consequently, the null hypothesis (Ho) was rejected. Figure 1 report the Hypothesis/Theories test done By HyperResearch SQA version 4.5.0

Figure 1: Hypothesis testing from the Research Interviews

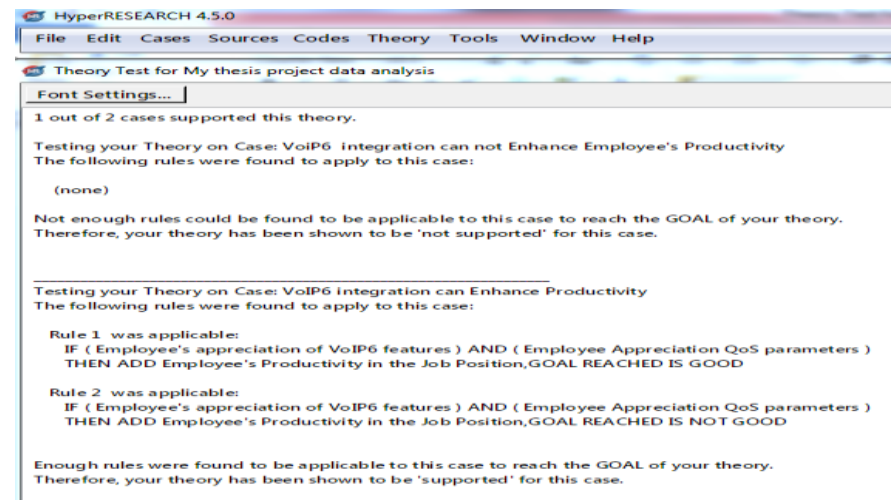

Validity and Reliability of the Research were done by ensuring that from the population of 150 workers, a reliable sample of 12 workers was defined and Data was collected from these people (8) who understand and apply notions of IP and VoIP and therefore could be of more impact in the research results. Besides, Data also was collected from direct people that can be a concern if the solution is implemented (4). Confidentiality of the interviewees and their sincere contribution was guaranty upon signature of a Concern form. Interviews were conducted by the researcher himself following an Interview Protocol Refinement (IPR) framework. The Data collection method was semi-structure.

\section{DATA ANALYSIS}

The frequency statistics of the analyses of the interview codes by HyperReserch software is given in Table 1 .

Table 1 FREQUENCY STATISTICS TABLEHYPERRESEARCH 4.5.0

\begin{tabular}{|l|l|l|l|l|l|l|}
\hline Codes & Total & Min & Max & Mean & $\begin{array}{l}\text { Std } \\
\text { Dev }\end{array}$ & Bar Graph \\
\hline $\begin{array}{l}\text { The domain of operation and } \\
\text { speciality of employees }\end{array}$ & 2 & 1 & 1 & 1 & 0 & \\
\hline $\begin{array}{l}\text { Employee Appreciation of the } \\
\text { PSTN and VoIP }\end{array}$ & 5 & 2 & 3 & 2.5 & 0.707 & \\
\hline $\begin{array}{l}\text { Employee Appreciation QoS } \\
\text { parameters }\end{array}$ & 3 & 1 & 2 & 1.5 & 0.707 & \\
\hline $\begin{array}{l}\text { Employee evaluation of internet } \\
\text { Protocols: IPv4 and IPv6 }\end{array}$ & 6 & 3 & 3 & 3 & 0 & \\
\hline $\begin{array}{l}\text { Employee's appreciation of the } \\
\text { Equipment's Capacities }\end{array}$ & 1 & 0 & 1 & 0.5 & 0.707 & \\
\hline $\begin{array}{l}\text { Employee's appreciation of VoIP6 } \\
\text { features }\end{array}$ & 1 & 0 & 1 & 0.5 & 0.707 & \\
\hline $\begin{array}{l}\text { Employee's Productivity in the Job } \\
\text { Position }\end{array}$ & 12 & 4 & 8 & 6 & 2.828 & \\
\hline
\end{tabular}


$66 \%$ of workers believe that VoIP in a Dual-Stack environment will constitute an effective alternative of the PSTN, therefore, ameliorating their productivity in their Job Position. $8 \%$ could give a tangible appreciation of VoIP6 features and effective appreciation of the equipment capacity of the existing IP data network. $25 \%$ effectively discussed on IPv4 versus IPv6. $50 \%$ contributed to appreciating the QoS parameters in consideration of the overall system response time and Delay Management. $25 \%$ could effectively compare features of PSTN and VoIP and therefore contributed to building more insight in their support of the proposed dualStack solution.

\section{EVALUATION OF THE EXISTING SYSTEM}

The IP data network of Camtel North-West is complex in architecture and divided according to the type of services (ToS) offered. Each type of service belongs to a separate management

domain organize more in a centralized than distributed way. Figure 2 shows the existing architecture.

Figure 2: EXISTING IPV4 DATA NETWORK ARCHITECTURE

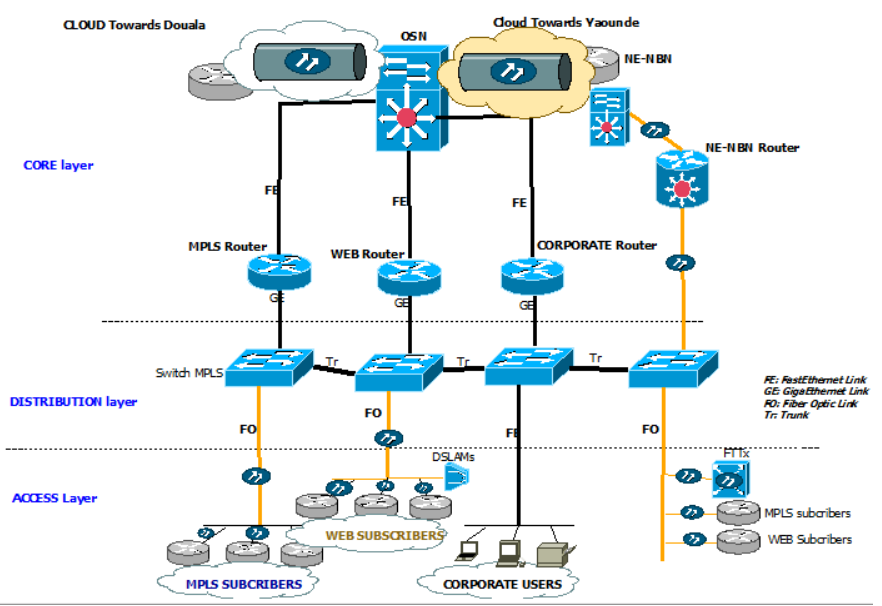

They are Three Management Domains:

The Corporate network Domain: use a Gateway Router (Corporate) to provide workers with corporate applications such as NGBSS (Next Generation Billing SubSystem), IMS (IP multimedia Services). Most of these corporate applications are accessible via web interfaces and workers login daily to do their jobs according to the profile classification. The access layer of this network domain is made of computers, phones and tablets using the corporate applications. The distribution level has switches interconnected to provide connectivity to these access devices. Also, the extension of this network is made with the use of a Wireless access point placed at the level of agencies. The Core level has a gateway router that faces the outbound link towards Yaounde through Bafoussam. This router is configured purposely to offer these corporate services and Internet connection to job positions.

The MPLS/VPN network Domain: this network domain is a dedicated network domain for organizations that need to interconnect their local or remote site using a secure VPN tunnel. The MPLS network provides IP forwarding based on labels, making remote sites to operate as if they were all locally connected. The access level of this network domain is made of subscriber's optical converters and routers that interface the MPLS router acting as a gateway for service provisioning and activation. The distribution layer implements segmentation of class of users by the configuration of VLANs. Each subscribers belonging to a particular VLAN. The Core level has an updated CISCO router of the 7600 series that acts as a gateway facing the outbound towards Douala. Service provided to the MPLS subscribers is from this Router that implement CEF (IP Cisco Express forwarding) with MPLS IP activated, and all $\mathrm{CR}$ (committed rate) bandwidth configurations. The main routing protocols implemented here are IS-IS (intermediate System-to-Intermediate System), BGP (Border Gateway Protocol), OSPF (Open Shortest Path) and RIP (Routing Information Protocol).

- The Internet network Domain: this is another network management domain that focuses on providing internet services to subscribers according to the technology used. xDSL subscribers, for example, will receive internet from DSLAM (Digital Subscriber Lines Access Multiplex) connected to the PSTN and the IP data network. Dedicated Subscribers will have broadband internet with the aid of the Internet Router that uses public IPs to face the outside. The access layer of the domain is therefore made of optical converters connected to the optical transmission networks (monomode / multimode fibres deployed) and routers of subscribers. The distribution layer has a backbone internet switch that centralized all connections of the Point of Presence. It implements VLANs segmentation attributed uniquely to each subscriber. The core layer has a backbone internet Router that makes use of complex IP configuration to provide internet to the various type of customers (LS, xDSL, Intern-link, Pop extension, etc.).

These three management domains are interconnected via the use of trunk links configured in manageable switches at the distribution level. All these three networks are implemented on a backbone made of Fibers links pointing to Douala and Yaoundé via Bafoussam. The traffic generated by the MPLS, Corporate and WEB Routers is forwarded out of the region through the Optical Switching Network (OSN) of the NFOB (National Fiber Optic Broadband) or the OSN NBN (National Broadband Network). The Voice communication is exclusively routed through the PSTN and all office workers communicate with each other either by the exchange of emails via the corporate network or exchange of calls through the Public Switched Telephone Network.

From the view of this architecture, the research question 1,2, and 3 required that we should define an input of integration. The Corporate Router happened to be the most suitable entry point. The Corporate Switch was used to create the required links with the VoIP Dual-Stack Gateway as shown in Figure 3. Figure 3: INTEGRATION POINT

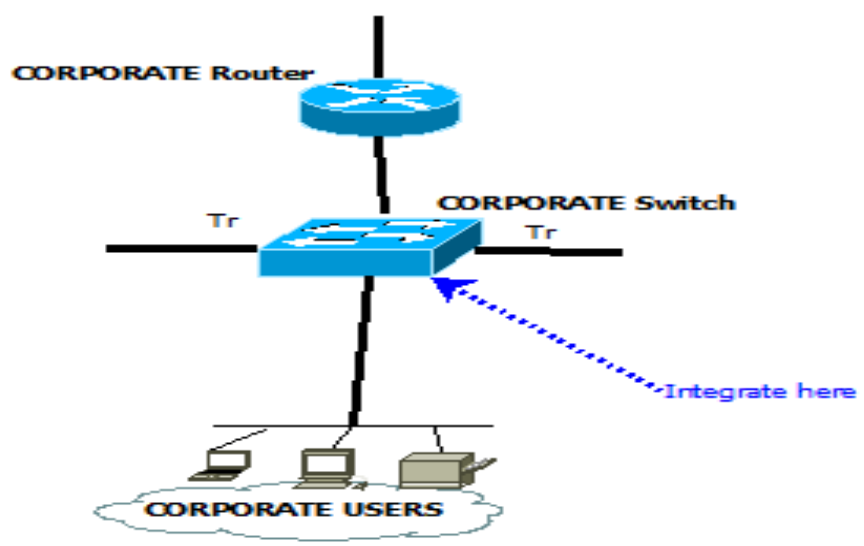




\section{VoIP DUAL-STACK DESIGN INTEGRATION}

Table2 below report on the various materials used to design the solution in Cisco Packet Tracer version 7.2. The goal here was to conceive the entire architecture on the simulation software by adding all network entities describes on Table 2, and connect all of them according to how that has to be done in case of physical implementation. It considers representing all network elements according to their function at each level of the network hierarchy (AL, DL, CL and AL).

Table 3 shows the specifications of the network design implemented in the simulation environment. It considers specifying the communication type, the mode of operation, and the main communication protocols with their related complementary protocols. It also shows the signaling protocols use in this environment with the type of CODEC required for compression of Voice signals.

Table 4 present the logical segmentation of IPv4 and IPv6 addresses. It also shows clear traffic separation of Voice and Data. IPhones and Softphones are attributed Numbers so that They can be able to call within the IPv4/IPv6 network domain. PSTN phones set also have attributed numbers. The logical IPv4/IPv6 segmentation creates separate subnet for VoIP users and Data Users. Such Separation helps in providing better flow management and security to traffic.

Table 2: MATERIALS USED IN THE SIMULATION ENVIRONMENT

\begin{tabular}{|c|c|c|}
\hline $\begin{array}{c}\begin{array}{c}\text { Network- } \\
\text { level }\end{array} \\
\end{array}$ & Material/equipment & Network operation/function \\
\hline \multirow{5}{*}{$\begin{array}{l}\text { Access Level } \\
\quad(\mathrm{AL})\end{array}$} & POTS Phones & Analogue phones connected to the PSTN \\
\hline & Cisco 8841 IP phones & $\begin{array}{c}\text { Provides VoIP connectivity with Dual-stack } \\
\text { compatibility (IPv4+IPv6). }\end{array}$ \\
\hline & Desktop/Laptops & $\begin{array}{l}\text { Used to run Cisco Softphones IP communicators } \\
\text { applications. TCP/IP Stack dual configured(IPv4/IPv6) }\end{array}$ \\
\hline & Hubs & $\begin{array}{l}\text { Provide Layer } 1 \text { connectivity to the VoIP Analog } \\
\text { Phones. Eliminate the need to connect PSTN lines } \\
\text { directly on the Core the element. Typically Layer } 1 \\
\text { functionalities }\end{array}$ \\
\hline & STP Cables & $\begin{array}{l}\text { Cat } 5 \mathrm{e} \text { Ethernet Cables used to establish physical links } \\
\text { between network entities at each level of the network } \\
\text { hierarchy. Switches->Hubs>IPhones->PCs }\end{array}$ \\
\hline $\begin{array}{l}\text { Distribution } \\
\text { Level (DL) }\end{array}$ & Cisco 2900s Switches & $\begin{array}{l}\text { Provides VLAN management and access to access } \\
\text { devices. Implement Quality Of Service (QoS)such as } \\
\text { queue support, 802.1q encapsulation fast link } \\
\text { convergence. It separates Voice and data traffic access. }\end{array}$ \\
\hline \multirow{3}{*}{$\begin{array}{l}\text { Core Level } \\
\quad(\mathrm{CL})\end{array}$} & WIFI AP & Provide VoIP access to Smart Phones and Tablets \\
\hline & $\begin{array}{l}\text { Cisco VG200 Router } \\
\text { Gateway }\end{array}$ & $\begin{array}{l}\text { Border router that aggregates multi-services(internet } \\
\text { and corporate applications) and implements traffic } \\
\text { classification, 802.1Q, traffic shaping and Link } \\
\text { efficiency. OSPFv3 is configured for convergence over } \\
\text { IPv6. It is incorporated behind the existing corporate } \\
\text { Router and linked to the NGN Soft switch towards } \\
\text { external VoIP users and Applications servers of the } \\
\text { IMS. }\end{array}$ \\
\hline & $\begin{array}{l}\text { Cisco } 1100 \text { ISR- } \\
\text { CUBE(Cisco Unified } \\
\text { Border Element Version } \\
12 \text { ) }\end{array}$ & $\begin{array}{l}\text { Media Gateway between the NGN Soft switch and the } \\
\text { IP data network. Implement all required config for } \\
\text { VoIP through NGN network( future research). Provide } \\
\text { SIP and H.323 session management for video and } \\
\text { audio system interconnection. }\end{array}$ \\
\hline $\begin{array}{l}\text { Service } \\
\text { Level(SL) }\end{array}$ & Future Research & $\begin{array}{c}\text { The researcher did not focus on this point, rather } \\
\text { consider this level for future development and } \\
\text { deployment towards remote sites. }\end{array}$ \\
\hline
\end{tabular}

TABLE 3: DESIGN PARAMETERS OF THE NETWORK

\begin{tabular}{|c|c|c|}
\hline Network Parameters & Description & Observations \\
\hline $\begin{array}{c}\text { Communication } \\
\text { network type }\end{array}$ & $\begin{array}{l}\text { Switched Communication } \\
\text { network }\end{array}$ & $\begin{array}{l}\text { Not Broadcast oriented except at Layer } 2 \text { of } \\
\text { the OSI Model }\end{array}$ \\
\hline Mode of Operation & $\begin{array}{c}\text { Packet-Switched } \\
\text { connectionless and } \\
\text { Connection-Oriented }\end{array}$ & $\begin{array}{c}\text { IP is basically Connectionless. When used } \\
\text { for circuit establishment of VoIP sessions, } \\
\text { virtual connection are required }\end{array}$ \\
\hline Logical topology & Star, P2P. & $\begin{array}{l}\text { Star at layer } 2 \text { to provide Multicast access to } \\
\text { access devices and P2P for outbound traffic } \\
\text { for WAN connectivity }\end{array}$ \\
\hline Type of link & $\begin{array}{l}\text { Fast Ethernet and Giga } \\
\text { Ethernet Link }\end{array}$ & $100 / 1000$ Mbits data rate \\
\hline $\begin{array}{l}\text { Main communication } \\
\text { Protocols }\end{array}$ & IPv4, IPv6 & Best-effort delivery \\
\hline
\end{tabular}

\begin{tabular}{|c|c|c|}
\hline $\begin{array}{l}\text { IP Telephony } \\
\text { Technology }\end{array}$ & VoIP & Digital technology \\
\hline $\begin{array}{l}\text { Complementary } \\
\text { Protocol }\end{array}$ & $\begin{array}{l}\text { Multicast IP, RTP, TCP, } \\
\text { DHCP6, DTP, OSPF, } \\
\text { OSPFv6, ICMP, ICMPv6 }\end{array}$ & $\begin{array}{l}\text { To provide real-time audio service and } \\
\text { connection-oriented sessions of terminals } \\
\text { belonging to different subnets with the } \\
\text { routing of Voice packets over IPv4 and } \\
\text { IPv6. }\end{array}$ \\
\hline Signalling Protocols & SCCP, H.323 & $\begin{array}{c}\text { Skinny Client Control Protocol, light } \\
\text { version of H.323 for voice session } \\
\text { management, connection and tearing down } \\
\text { of sessions }\end{array}$ \\
\hline $\begin{array}{c}\text { Standard } \\
\text { Encapsulation }\end{array}$ & IEEE 802.1q (Dot1.q) & VLAN tagging \\
\hline Service Provided & $\begin{array}{l}\text { IP telephony over } \\
\text { IPv4/IPv6 }\end{array}$ & $\begin{array}{l}\text { Cisco IP telephony service configured with } \\
\text { activation of Dial Per numbers. }\end{array}$ \\
\hline $\begin{array}{c}\text { Number of network } \\
\text { elements at the Access } \\
\text { level }\end{array}$ & 36 & $\begin{array}{l}\text { Comprises IP phones, Softphones, Mobile } \\
\text { Tablet, Desktops, Laptops }\end{array}$ \\
\hline $\begin{array}{l}\text { Number of network } \\
\text { element at the } \\
\text { Distribution level }\end{array}$ & 5 & $\begin{array}{c}\text { Switches configured to provide VLAN } \\
\text { segmentation, connection to access devices } \\
\text { and forwarding of Voice and data packets } \\
\text { for routing }\end{array}$ \\
\hline $\begin{array}{c}\text { Number of Network } \\
\text { elements at the Core } \\
\text { level }\end{array}$ & $\begin{array}{l}5 \text { routers, } 2 \text { layers } 3 \\
\text { switches and one Server. }\end{array}$ & $\begin{array}{l}\text { The routers provide IP telephony service and } \\
\text { traffic routing with QoS policy class. }\end{array}$ \\
\hline CODEC & G.729 & To minimize bandwidth consumption \\
\hline QoS Management & $\begin{array}{c}\text { Traffic prioritization, Class } \\
\text { of Service }(\mathrm{CoS})\end{array}$ & $\begin{array}{c}\text { Minimization of delays and optimization of } \\
\text { traffic }\end{array}$ \\
\hline $\begin{array}{c}\text { Average call } \\
\text { connecting time }\end{array}$ & $9 \mathrm{~ms}$ & Acceptable \\
\hline $\begin{array}{l}\text { Average system } \\
\text { response time }\end{array}$ & $8 \mathrm{~ms}$ & Acceptable \\
\hline Simulation Duration & $30 \mathrm{~min}$ & $\begin{array}{l}\text { The testing procedure and confirmation of } \\
\text { telephony service availability in the inter- } \\
\text { network domain }\end{array}$ \\
\hline
\end{tabular}

\begin{tabular}{|c|c|c|c|c|c|c|}
\hline \multirow[t]{2}{*}{$\begin{array}{l}\text { Logical } \\
\text { Domain }\end{array}$} & \multirow[t]{2}{*}{ SITES } & \multicolumn{3}{|c|}{ IP SUBNET /CIDR } & \multicolumn{2}{|c|}{$\begin{array}{c}\text { VLAN } \\
\text { segment }\end{array}$} \\
\hline & & & VoIP & DATA & $\begin{array}{c}\text { Dat } \\
\text { a }\end{array}$ & $\begin{array}{c}\text { VoI } \\
\mathrm{P}\end{array}$ \\
\hline \multirow[t]{5}{*}{$\begin{array}{c}\text { IPv4 } \\
\text { Subnetting }\end{array}$} & $\begin{array}{c}\text { Bamend } \\
\text { a }\end{array}$ & \multicolumn{2}{|c|}{$192.168 .32 .0 / 19$} & $192.168 .0 .0 / 19$ & 36 & 10 \\
\hline & Kumbo & \multicolumn{2}{|c|}{ 192.168.128.0/19 } & $192.168 .64 .0 / 19$ & 37 & 11 \\
\hline & Nkambe & \multicolumn{2}{|c|}{$192.168 .160 .0 / 19$} & $192.168 .224 .0 / 19$ & 38 & 12 \\
\hline & Wum & \multicolumn{2}{|c|}{$192.168 .96 .0 / 19$} & $192.168 .192 .0 / 19$ & 39 & 13 \\
\hline & Bambili & \multicolumn{2}{|c|}{$172.24 .200 .62 / 27$} & $172.24 .200 .0 / 27$ & 40 & 14 \\
\hline \multirow{6}{*}{$\begin{array}{c}\text { IPv6 } \\
\text { Subnetting }\end{array}$} & & \multirow{2}{*}{\multicolumn{2}{|c|}{ FC00:0:0:200::/56 }} & \multirow[b]{2}{*}{ FC00:0:0:100::/56 } & & \\
\hline & $\begin{array}{c}\text { Bamend } \\
\mathrm{a}\end{array}$ & & & & 36 & 10 \\
\hline & Kumbo & \multicolumn{2}{|c|}{ FC00:0:0:700::/56 } & FC00:0:0:600::/56 & 37 & 11 \\
\hline & Nkambe & \multicolumn{2}{|c|}{ FC00:0:0:800::/56 } & FC00:0:0:900::/56 & 12 & 38 \\
\hline & Wum & \multicolumn{2}{|c|}{$\begin{array}{c}\text { FC00:0:0:3000::/5 } \\
6\end{array}$} & $\begin{array}{c}\text { FC00:0:0:4000::/5 } \\
6\end{array}$ & 39 & 13 \\
\hline & Bambili & \multicolumn{2}{|c|}{$\begin{array}{c}\text { FC00:0:0:1000::/5 } \\
6\end{array}$} & $\begin{array}{c}\text { FC00:0:0:2000::/5 } \\
6 \\
\end{array}$ & 40 & 14 \\
\hline \multicolumn{7}{|c|}{ IPv4 /IPv6 Inter-LINK } \\
\hline \multicolumn{4}{|c|}{ IPv4 } & \multicolumn{3}{|c|}{ IPv6 } \\
\hline $\begin{array}{l}\text { Corporate- } \\
\text { ITS- } \\
\text { KUMBO } \\
\text { BRAS }\end{array}$ & \multicolumn{3}{|c|}{$197.159 .8 .4 / 30$} & \multicolumn{3}{|c|}{ 2001:0:0:200::/64 } \\
\hline $\begin{array}{c}\text { Corporate- } \\
\text { ITS- } \\
\text { NKAMBE } \\
\text { BRAS }\end{array}$ & \multicolumn{3}{|c|}{$197.159 .8 .8 / 30$} & \multicolumn{3}{|c|}{ 2001:0:0:300::/64 } \\
\hline $\begin{array}{c}\text { Corporate- } \\
\text { ITS-WUM } \\
\text { BRAS }\end{array}$ & \multicolumn{3}{|c|}{$197.159 .8 .16 / 30$} & \multicolumn{3}{|c|}{$2001: 0: 0: 500:: / 64$} \\
\hline $\begin{array}{l}\text { Corporate- } \\
\text { ITS- } \\
\text { BAMBILI } \\
\text { BRAS } \\
\end{array}$ & & .159. & $12 / 30$ & 2001:0:0:4 & $\because: / 64$ & \\
\hline $\begin{array}{l}\text { Corporate- } \\
\text { ITS-UBE }\end{array}$ & & 24.19 & $.0 / 30$ & 2001:0:0:6 & $:: / 64$ & \\
\hline UBE-NGN & & .24 .1 & $.4 / 30$ & 2001:0:0:7 & $:: / 64$ & \\
\hline $\begin{array}{l}\text { Routing } \\
\text { Protocol }\end{array}$ & & SPF, & GP & OSPFv3, M & i-BGP & \\
\hline IP service & & & IP telep & ony service & & \\
\hline $\begin{array}{c}\text { Layer } 2 \\
\text { Protocol } \\
\end{array}$ & & & & $2.1 \mathrm{q}$ & & \\
\hline QoS & & & $\mathrm{CoS}, \mathrm{T}$ & gging, mls & & \\
\hline Signalling & & & & SCCP & & \\
\hline Security & & & Port securi & , Mac Binding & & \\
\hline & & ORK & RS IP PHONE & NUMBERS & & \\
\hline $\begin{array}{c}\text { WORKER } \\
\text { POSITIO } \\
\mathbf{N}\end{array}$ & $\mathbf{L O C A}^{\prime}$ & & $\begin{array}{l}\text { IP PHONI } \\
\text { NUMBER }\end{array}$ & \begin{tabular}{l|l} 
PSTN N & P \\
\end{tabular} & MBER & \\
\hline SFR & BAME & & 1000 & 2333 & & \\
\hline SCS & BAME & & 1003 & 2333 & 015 & \\
\hline $\mathbf{R R}$ & BAME & & 1001,1024 & 2333 & & \\
\hline SCO & BAME & & 1025 & 2333 & & \\
\hline SEC.RR & BAME & & 1026,1034 & 2333 & 940 & \\
\hline
\end{tabular}




\begin{tabular}{|c|c|c|c|}
\hline $\begin{array}{c}\text { AC } \\
\text { KUMBO }\end{array}$ & KUMBO & 2000,2020 & NONE \\
\hline $\begin{array}{c}\text { PC } \\
\text { NKAMBE }\end{array}$ & NKAMBE & 2001,2021 & NONE \\
\hline PC WUM & WUM & NONE & 233343520 \\
\hline
\end{tabular}

\section{IMPLEMENTATION}

The various parameters defined in Table 2 and Table 3 were used to implement the Dual-Stack VoIP solution. Figure 4 shows the Physical Topology of the Solution. IP telephony service was implemented in the Corporate Router to provide Voice services to users (SFR: Service Finance and recovery, SCS: Service Control and Security, RR: Regional Representative, SCO: Service Commercial, AC: Commercial Agency, PC: Commercial Point with extension towards remote site within the same management domain - Kumbo, NKAMBE, WUM, BAMBILI). Depending on the sensitivity of the job position, the user may have a softphone, a VoIP phone and the traditional PSTN set (example the SCS who has Softphone: Number 1023, IP phone Number 1003 and PSTN set Number 233361015).

Figure 4: IMPLEMENTATION OF VOIP DUAL-STACK CONFIGURATION

Cisco Packet Tracer - EIPROJET MASTER ICT-UMMY PROJECT THESISSintegration VolP, pht File Edt Optons View Took Extensions Heb

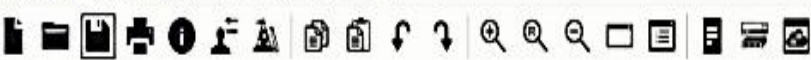

IPv4 data traffic with VLAN tagging 36 (IPv6 subnet FC00:0:0:200::/56 for Voice and subnet FC00:0:0:100::/56 for

Figure 5: HOST DUAL-STACK CONFIG.

Data), and IPv4 Voice traffic using IP phone 1000, with VLAN tagging 10. The 233361000 number will be used by SFR to call IP phone 1000 and any other number within the intra-network domain.

- Testing IPv4 reachability in the Inter-VLAN

Figure 6: IPv4/IPv6 INTER-VLAN REACHABILITY

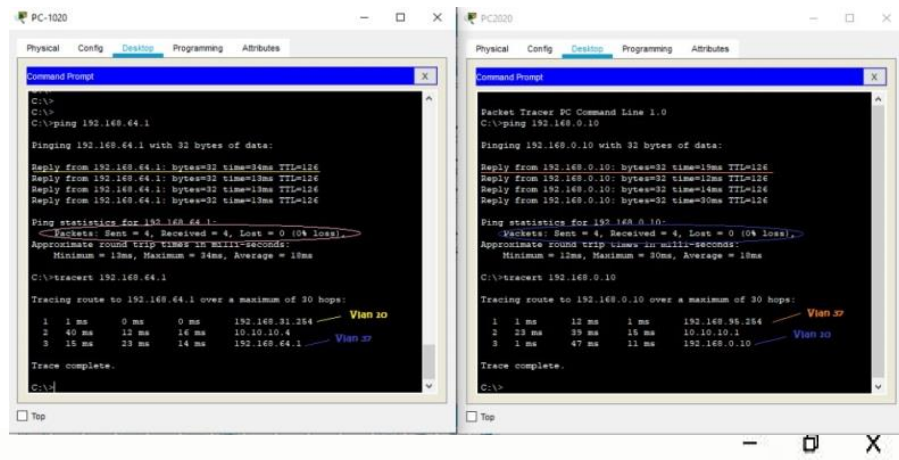

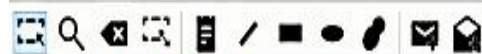

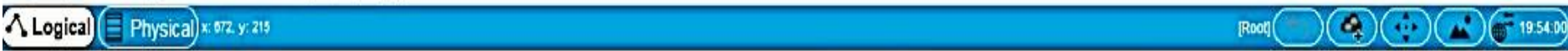

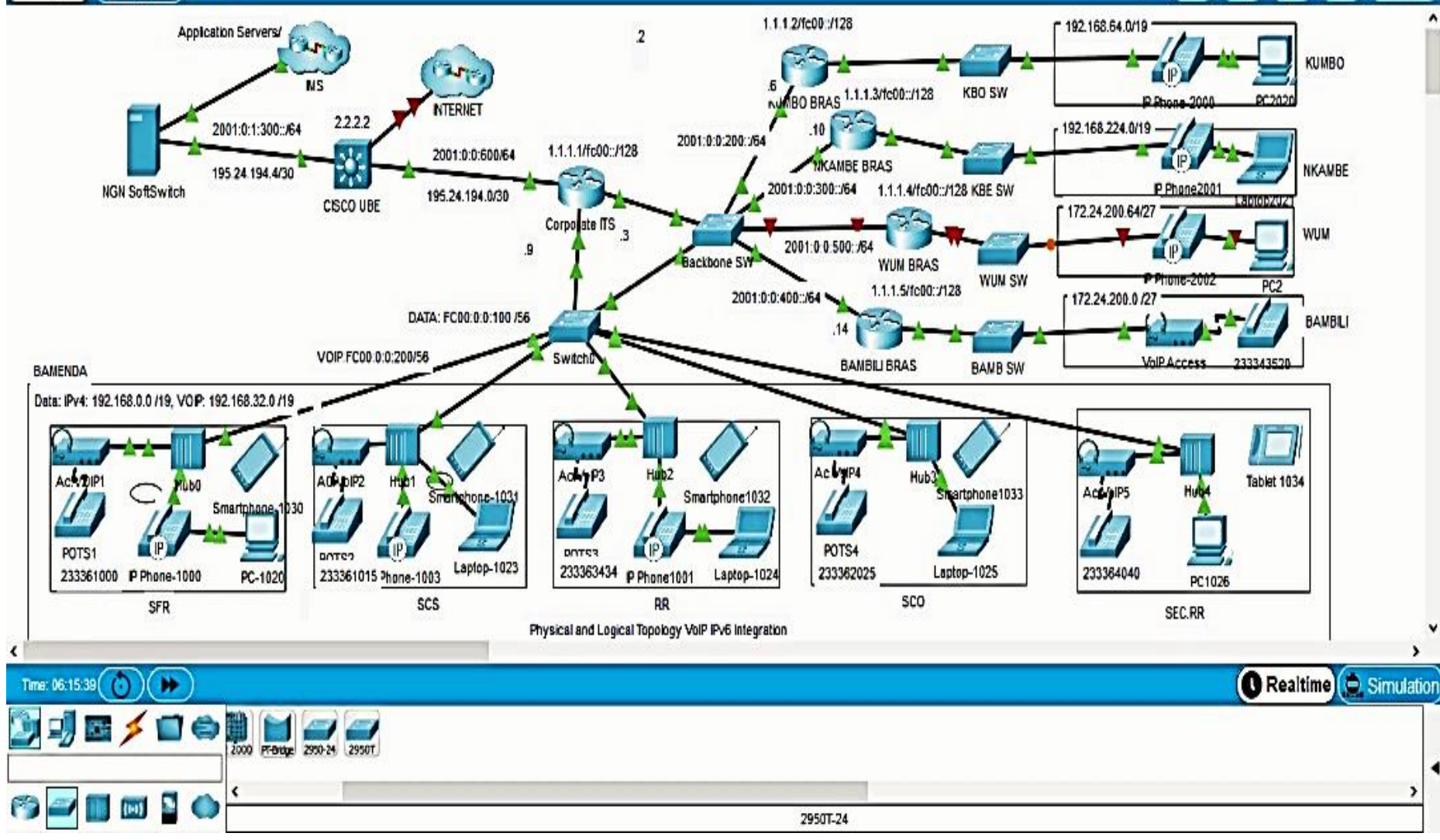

\section{TESTING}

\section{- Dual-Stack Configuration of a Host}

PC1020 belonging to SFR received IPv4 and IPv6 addresses from the DHCP pool 192.168.0.0/19 with default gateway 192.168.31.254, and, DHCPv6 pool FC00:0:0:100::/56 with default gateway FC00::100:192:168:31:254. PC1020 forward
Inter-Vlan Hosts between Vlan 10-192.168.0.0/19 in Bamenda and Vlan 37 192.168.128.0/19 in Kumbo. It means a terminal belonging to subnet 192.168.32.0/19 (FC00:0:0:200::/56) can call another terminal 192.168.128.0/19 over IPv4 or FC00:0:0:700::/56 over IPv6.

Reachability within these subnets is confirmed when Terminals receive IP addresses and mapped accordingly with 
MAC addresses of terminals. The Corporate IP Telephony Service (ITS) which is the VoIP router gateway that acts as the Call Manager is successfully activated and attributes the Phone number according to the register ephone in the directory.

Figure 7: Host Dual-Stack Configuration

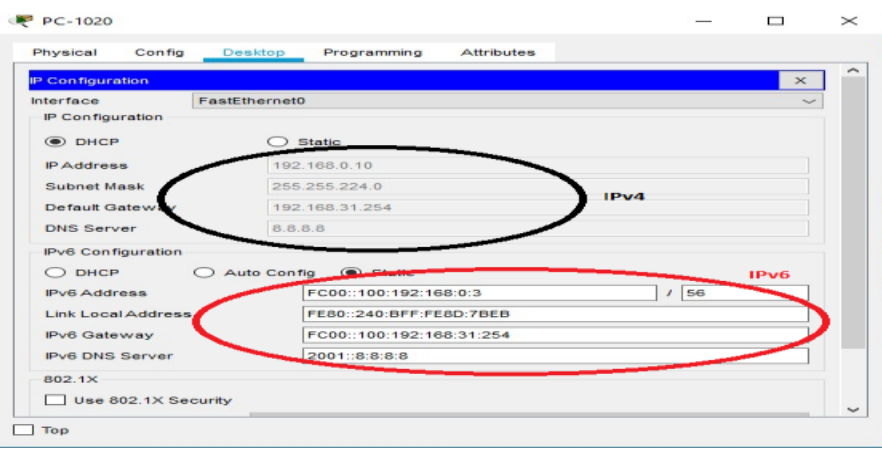

over 3 hops using OSPFv3 to reach a host in Kumbo under VLAN tagging 37.

\section{- Testing Reachability between IPhones and PSTN phones}

POTS phone with number 233361000 made a call towards an IP phone with a number 2000 found in a remote site at KUMBO and belonging to subnet 192.168.64.0 / FC00:0:0:700:: on Vlan 11. The researcher could observe the 2000 IP phone set rang. This means that if with the SCCP protocol, the analogue phone can communicate with IP phone over IPv4/IPv6 protocol, then it can be more effective when using call manager running SIP and communicating with SS7 on the

PSTN side and H.323 protocol on the IP side. Also, voice traffic forwarded on dual interfaces confirms that VoIP can

Figure 8: IP Phone FULL OPERATION

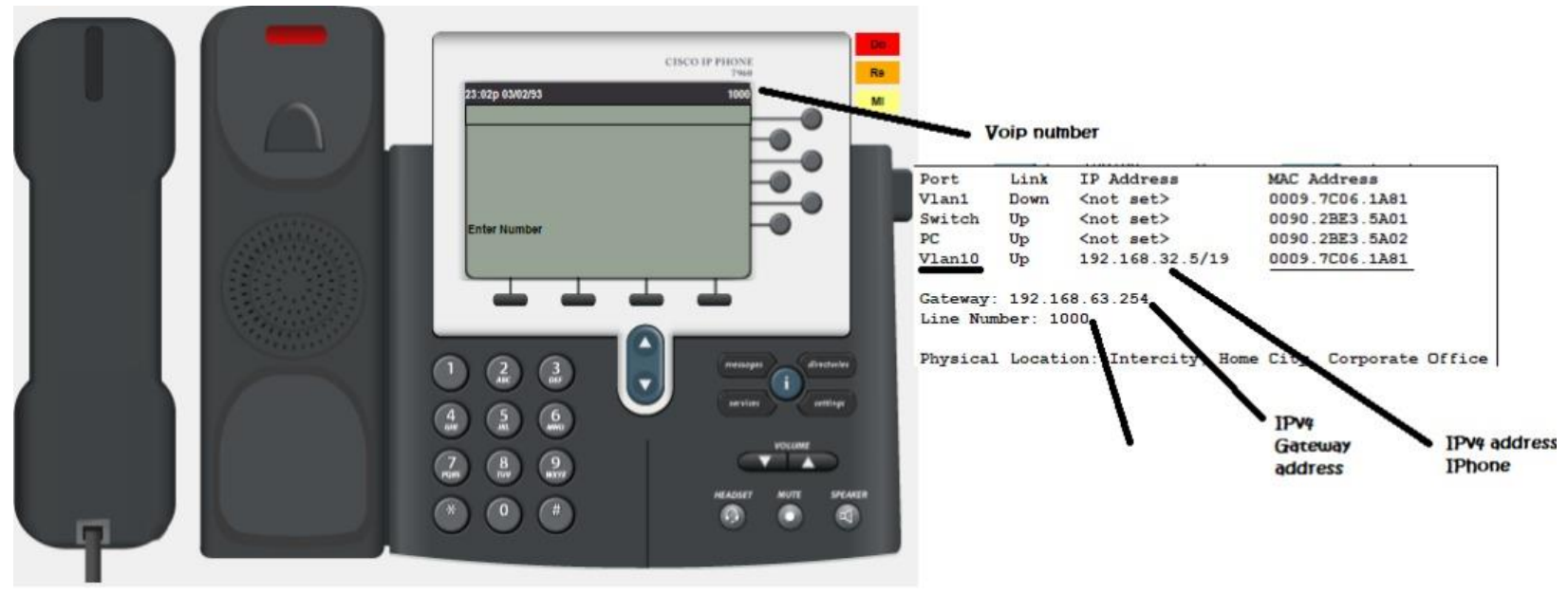

The Dial tone is provided by the SCCP (Skinny Call Control Protocol also known as Signaling Connection Control Part), a Cisco communication protocol, which is a small version of the H.323 protocol responsible in call settings over IP through TCP port 2000. A fully operational IP phone is shown in Figure 7.

\section{- Testing IPv6 Reachability}

Figure 8: Tracing IPv6 Traffic

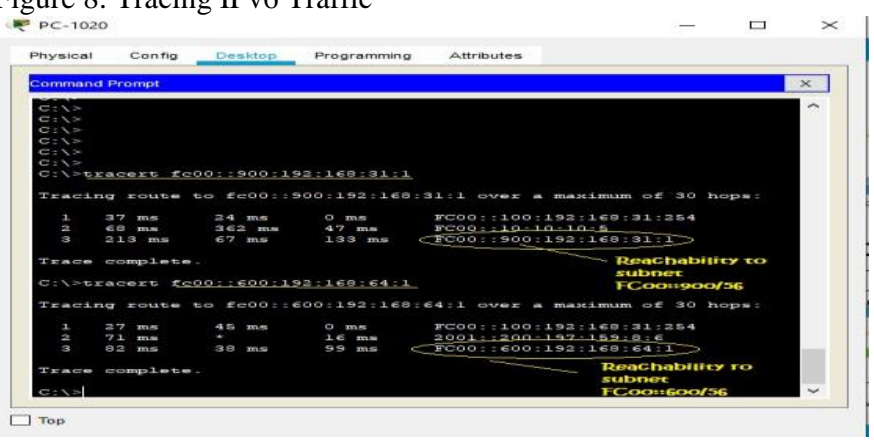

To confirm the proper reachability, the researcher conducted a Traceroute from a host to a subnet FC00:0:0:900::/56 and to a host in subnet FC00:0:0:600::/56 via the Corporate ITS gateway configure with a global public gateway 2001::200:197:159:8:6. The researcher could see that the host FC00::900:192:132:31:1 belonging to VLAN 12 in Nkambe could be reached after 3 hops. Also, IPv6 packets were routed
Figure 9: Inter-routing PSTN and IP calls

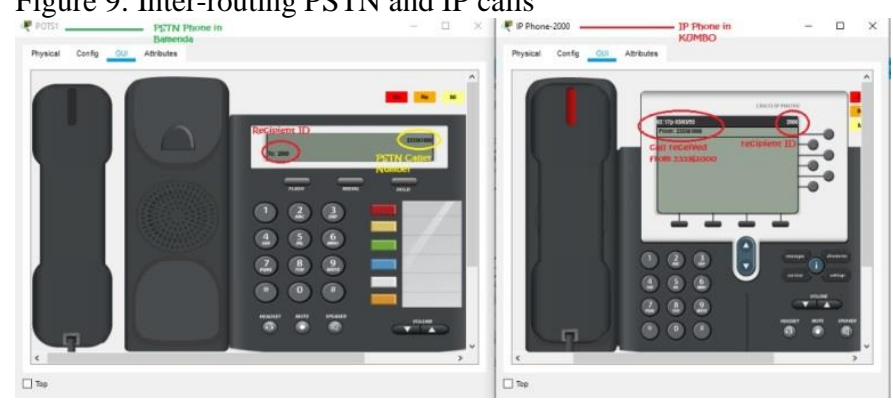

operate over IPv6 without affecting the data services running over IPv4.

\section{- $\quad$ Testing QoS parameters}

The testing of Quality of Service parameters can be categorized into three groups.

$>\quad$ The first is a test related to Timeliness where parameters such as delay, Jitter and response time are tested. Due to the simulation environment, the researcher could not be pronounced on the system response time. But delay particularly was monitored in term of appreciating the time taking for the gateway to attribute a tone and the time the called phone takes to ring during a calling session. It was satisfactory. Jitter here was not observed.

$>\quad$ The second is a test related to Bandwidth where parameters such as Systems-level data rate, Application-level data rate and Transaction time. Here, the used of 100/1000 
Mbits links and interfaces ensures us sufficient bandwidth for call Voice and data transfer. A further test could be conducted by saturating the link with overflow traffic and observing Call quality.

$>\quad$ The third is a test related to Reliability where parameters such as packet loss rate, system response time, Mean time to failure (MTTF), Mean time to repair (MTTR, Mean time between failures (MTBF), and Percentage of time available. At this point, the researcher focus on observing that packets loss rate is less than $1 \%$.

The effectiveness of such tests can be done by using and modifying the normal behaviour of some QoS traffic management processes such as Classification (attribution of certain parameters to packet headers to generate a specific class of traffic; the research implement this in switches to apply special consideration on voice packet in the voice Vlans- CoS mls), Queuing (cache of packets to regulate bandwidth occupation and processing), and, utilizing specialized software like ETHEREAL network monitoring for collection of a packet to analyze (packet actual arrival time and the estimated arrival time to find delay or the differences in the inter-arrival time of the RTP packets to calculate Jitter variation). OPNET software will contribute greatly with a graphical representation of traffic where these QoS parameters can be identified and analyze. However, the research objectives do not address this aspect where QoS parameters are specifically studied and analyze; rather focus in providing an integration solution, an alternative to an existing voice communication system and ensuring effective connectivity of calls within the IPv4/IPv6 inter-network domain. The use of network tools such as Ping or Traceroute helped the researcher in the simulation environment to test connectivity, response time and packet loss rate between end devices. The purpose of optimization of such a solution (for the amelioration of the quality of voice by minimizing latency, Jitter, packet loss) once implemented can therefore give room to further research that focuses in evaluating VoIP performance and how to improve it in the Camtel IP data network. The research, nevertheless, gave special attention in ensuring that the system response time or latency should be less than $20 \mathrm{~ms}$ while keeping packet loss rate to less than $1 \%$. Just these two considerations in the simulation platform help to enhance connectivity and call routing between VoIP terminals as it is recommended for implementation of a VoIP system.

\section{CONCLUSION}

The PSTN and the IP telephony Network are two different platforms that provide almost the same type of services. The limitations related to analogue systems may contribute to believing that so far IP telephony is built over digitalized network entities, it is the best compare to the PSTN and the services this network can offer. However, this research did not intends to evaluate deeply which of them is best, but rather emphasize on benefit provides by IP telephony and show how this can contribute to ameliorate workers communication potentiality.

After putting in place a simulation environment, architecture was developed whereby all required network entities were put in place to come out with a design that can be close enough to reality. The findings related to the physical requirements shows that the need to provide links that have sufficient bandwidth is real. The logical requirement shows that separation of voice and data traffic and application of traffic prioritization is one of the fundamental conditions if we must achieve a good QoS both for Data and Voice traffic. The proper configuration of network entities at all level of the network hierarchy is very important and none is to be neglected. IPv4 and IPv6 can successfully operate simultaneously and the integration of new services can be done so far a good logical plan of the network is conducted. It means segmenting users by functions and type of service and applying proper addressing on network entities. When the integration design is well-conceived, a regular analogue phone can forward voice signal to a digitalized network and successfully place calls that can connect to the IP phones. Also, telephony applications such as Softphones that are connected to the IPv4/IPv6 network can connect to the PSTN and exchange voice signal with POTS phones. VoIP happened to be effective according to this simulation and convincing enough to act as an alternative solution to the traditional PSTN. It is all about finding optimal integration design and applying them following standard and respect of requirements that if neglected, can affect the overall system once bypassed such has integrating applications that are delay-sensitive with applications that are demanding in term of resources consumption causing delays (propagation and transmission) and bandwidth saturation. The observation of IPv6 operation reassures the researcher in term of better throughput compares to the IPv4. The flexibility in which the various phones can easily communicate with each also confirms that this solution can contribute to enhancing workers' productivity.

\section{REFERENCES}

1. Kevin Macgregor, Global Best Practice: An introduction to Service Integration and Management and ITIL, White paper, January 2015, page 3, 5.

2. Farrukh Saleem, Enterprise application integration as a middleware: Modification in data \& process layerB Conference: 2014 Science and Information Conference (SAI), abstract, August 2014.

3. R. Gilligan, E. Nordmark, RFC 1933: Transition Mechanisms for IPv6 Hosts and Routers, April 1996.

4. PowerNext, Powering the next Market, permanent contract, November 2016, page 1-2.

5. James Finister, Kevin Holland,An introduction to service Integration and Management and ITIL, AXELOS, Global Best Practice, White paper, January 2015, page 5, 7

6. Mihaela Van Der Schaar, Philip A.Chou, Compression, Networking and Systems: Multimedia Over IP and Wireless systems, 2007, Elsevier Inc,

7. Jarvin Technologies, Network Protocols Handbook, $2^{\text {nd }}$ edition, 2004.

8. Aircall, Is VoIP a reliable Service for your Business? 2020.

9. Andrew G. Blank, TCP/IP foundation; San Francisco London, SYBEX Inc, 2004

10. Auben networks, IPv6 Migration techniques, Networking for people, 2014.

11. Briefing paper Internet Society, "IPv6: Why and how government should be involved" in June 2009

12. Cameron Johnson, Nextiva, Is VoIP secure? The ultimate guide to VoIP security and Call encryption, blog, 2020.

13. Farrukh Saleem, Enterprise application integration as a middleware: Modification in data \& process layerB Conference: 2014 Science and Information Conference (SAI), abstract, August 2014

14. James Finister, Kevin Holland, An introduction to service Integration and Management and ITIL, AXELOS, Global Best Practice, White paper, January 2015 , page 5,7

15. Gao Xianrui / Zhou Yanqing, the NGN age has arrived, Huawei technologies, April 2005

16. Jaydip Sen, Convergence Next-Generation Networks, Indian Statistical Institute, Kolkata, INDIA, June 2014 
17. John G. Proakis, Rolger L Freeman, Fundamentals of Telecommunications, $2^{\text {nd }}$ Edition Willey Interscience, 2005.

18. JPNIC, Analysis and Recommendations on the Exhaustion of IPv4 Address Space, Expert Research Team on Number Resources Utilization, March 24, 2006.

19. Kirian MakhiJani, Challenge for IP 2020, Senior Research Scientist, Future Networks Huawei Technologies, US Research Center-CA, Huawei. July 2016, page 1-37.

20. PowerNext, Powering the next Market, permanent contract, November 2016, page 1-2.

21. Practical UNIX and Internet security, TCP/IP networks, IPv4: the internet protocol version 4, 1999.

22. R. Gilligan, E. Nordmark, RFC 1933: Transition Mechanisms for IPv6 Hosts and Routers, April 1996.

23. R. Hinden, J. Postel, RFC 1897: IPv6 Testing Address Allocation, January 1996, page 227-2.

24. Wallingford Theodore, Switching to VoIP, O’Reilly Media Inc, 2005, page 1

25. Floriano De Rango, Mauro Tropea, Peppino Fazio, Multimedia Traffic Over wireless and Satellite Networks, DEIS Dept., University of Calabria, Italy, ISBN 978-953-7619-70-1, pp 500, February 2010.

26. Peter L Dordal, An introduction to Computer networking, Release 2.0.2, 2020, http://intronetworks.cs.luc.edu/current/ComputerNetworks.pdf

27. Peter Dell, On the Dual-Stacking Transition to IPv6: A forlon Hope, Curtin University, 2018, https://doi.org/10.1016/j.telpol.2018.04.005

28. Nick Buraglio, Three reasons why IPv6 is worth the effort, GO IPv6, APNIC, blog 2018, https://blog.apnic.net/2018/12/13/three-reasonswhy-ipv6-is-worth-the-effort/.
29. Molay Ghost, Reliance Jio boots India past $20 \%$ IPv6 capability, Tech matters-https://blog.apnic.net/2017/02/07/, 2017.

30. CISCO IT, IPv6 implementation, CISCO, Document ID:1488429535478851,

2013 https://www.cisco.com/c/en/us/solutions/collateral/enterprise/cisco -on-cisco/IPv6-Implementation_CS.html.

31. Charles Petrie, Olivier Spatscheck, future Internet protocols, IEEE, IEEE Internet Computing (Volume: 16, Issue: 6, Nov.Dec. 2012 ), page 11-13.

32. Andrew Kirch, IPv6, Let the Sky Fall. Zenoss Own IT, Zenoss Cloud, 2015. https://www.zenoss.com/blog/ipv6-let-the-sky-fall.

33. Wikiwand, Packet Switching, wikepedia, the free encyclopedia, 2019 https://www.wikiwand.com/en/Packet_switching.

34. Lian, Liu, Hou, Zou Yang, ACTA SEISMOLOGICA SINICA, Application of IPv6 and NGN to the information network of earthquake preparedness and disaster mitigation, doi: 10.1007/s11589-008-0665-7, November 2008.

35. Daniel Noworatzki, TeleDynamics Think Tank How IPv6 Benefits VoIP, TELEynamic, 2019

36. Finnel K., PSTN Vs VoIP: What's best for your business ? 2018 https://searchunifiedcommunications.techtarget.com/feature/PSTN -vs-VoIP-Whats-best-for-your-business.

37. Unuth Nadeem, What is VoIP and can it be reduced? Dealing with echoes and overlapping noises in VoIP calls., 2019. https://www.lifewire.com/what-is-latency-and-how-it-can-bereduced-3426314 